\title{
A New 2 + 1-Dimensional Integrable Variable Coefficient Toda Equation
}

\author{
Yanan Huang1, Junhong $\mathrm{Yao}^{2}$, Ting Su${ }^{2 *}$ \\ ${ }^{1}$ Beijing Institute of Technology, Zhuhai Campus, Zhuhai, China \\ ${ }^{2}$ College of Science, Henan University of Engineering, Zhengzhou, China \\ Email: ^suting1976@163.com
}

How to cite this paper: Huang, Y.N., Yao, J.H. and Su, T. (2021) A New $2+$ 1-Dimensional Integrable Variable Coefficient Toda Equation. Journal of Applied Mathematics and Physics, 9, 2152-2158.

https://doi.org/10.4236/jamp.2021.98134

Received: July 30, 2021

Accepted: August 28, 2021

Published: August 31, 2021

Copyright $\odot 2021$ by author(s) and Scientific Research Publishing Inc. This work is licensed under the Creative Commons Attribution International License (CC BY 4.0).

http://creativecommons.org/licenses/by/4.0/

\begin{abstract}
In this paper, a new integrable variable coefficient Toda equation is proposed by utilizing a generalized version of the dressing method. At the same time, we derive the Lax pair of the new integrable variable coefficient Toda equation. The compatibility condition is given, which insures that the new Toda equation is integrable. To further analyze the character of the Toda equation, we derive one soliton solution of the obtained Toda equation by using separation of variables.
\end{abstract}

\section{Keywords}

The Generalized Dressing Method, Variable Coefficient Toda, Separation of Variables

\section{Introduction}

Integrable variable coefficient equations describe the real world in many fields of physical and engineering sciences. Many researchers are devoted to discussing these equations by utilizing different methods ref. [1]-[6]. In ref. [7] [8], Dai and Jeffrey extended the dressing method to a generalized version for solving nonlinear evolution equations associated with matrix spectral problems and variable coefficient cases, in which a key is that variable coefficient dressing operators are transformed to different variable coefficient ones. By using the generalization, we have studied integrable variable coefficient coupled Hirota equation in ref. [9]. In ref. [10] [11], integrable variable coefficient Manakov model and cylindrical NLS equation are discussed in detailed, respectively. In ref. [12], we developed the generalized dressing method to the discrete system and an integrable variable coefficient Toda equation is researched. Recently, the dressing method 
is extended to a matrix Lax pair for Camassa-Holm equation in ref. [13], in which interactions between soliton and cuspon solutions of the system are studied. The dressing method as nonlinear superposition in Sigma models has been researched by Dimitrios Katsinis et al. in ref. [14]. Multi-lump solutions of KP equation with integrable boundary are discussed in ref. [15] by using the generalized dressing method. Nabelek et al. in ref. [16] studied Kaup-Broer system and derived its solutions.

In the present paper, we extend the generalized dressing method to discrete operators similar to ref. [12]. Through direct calculations, we derive a new integrable variable coefficient Toda equation

$$
\chi_{y y}-\chi_{n, t t}-\Delta n(n-1)\left(\mathrm{e}^{\chi_{n-1}-\chi_{n}}-1\right)=0
$$

where, the coefficient is related to $n, \Delta=E-E^{-1}$. Equation (1.1) is an extension of the well known two-dimensional Toda equation. We will construct one soliton solution of (1.1).

The present paper is organized as follows. In Section 2, we obtain a new integrable variable coefficient Toda equation based on the generalized dressing method. In Section 3, as an application, we derive one soliton solution of (1.1) by utilizing the separation of variables.

\section{Integrable Variable Coefficient Toda Equation}

In this section, we first summarize the variable coefficient version of the dressing method. We extend the generalized version of the dressing method to discrete systems and derive different integrable cylindrical Toda lattice equations by choosing different operators.

First, we consider three linear differential difference operators ref. [12]

$$
\begin{aligned}
& \mathbf{F}(n, m, t, y) \psi_{n}=\sum_{m=-\infty}^{\infty} F(n, m, t, y) \psi_{m}, \\
& \mathbf{K}_{+}(n, m, t, y) \psi_{n}=\sum_{m=n}^{\infty} K_{+}(n, m, t, y) \psi_{m}, \\
& \mathbf{K}_{-}(n, m, t, y) \psi_{n}=\sum_{m=-\infty}^{n} K_{-}(n, m, t, y) \psi_{m} .
\end{aligned}
$$

Similar to the generalized dressing method application to continuous system, we introduce the triangular factorization about the operator " $\mathbf{F}$ ”

$$
\mathbf{I}+\mathbf{F}=\left(\mathbf{I}+\mathbf{K}_{+}\right)^{-1}\left(\mathbf{I}+\mathbf{K}_{-}\right),
$$

where $\mathbf{I}$ is the identity operator, $\mathbf{K}_{+}(n, m, t, y)=0$ for $m<n$ and $\mathbf{K}_{-}(n, m, t, y)=0$ for $m>n$. It is assumed that

$$
\sup \sum_{m=n_{0}}^{\infty}\left|K_{ \pm}(n, m, t, y)\right| \psi_{m}<\infty, \quad \sup \sum_{m=n_{0}}^{\infty}|F(n, m, t, y)| \psi_{m}<\infty \text {, }
$$

for all $n_{0}>-\infty$. For convenience, we denote $F(n, m, t, y)=F(n, m)$, $K_{ \pm}(n, m, t, y)=K_{ \pm}(n, m)$. The discrete Gelfand-Levitan-Marchenko (GLM) equation can be obtained from (2.2), which reads in ref. [12] 


$$
F(n, m)+K_{+}(n, m)+\sum_{s=n}^{\infty} K_{+}(n, s) F(s, m)=0 .
$$

We introduce two differential-difference operators $\mathbf{M}_{1}$ and $\mathbf{M}_{2}$ defined by

$$
\begin{gathered}
\mathbf{M}_{1}=\partial_{t}+\partial_{y}-n \mathbf{E}, \\
\mathbf{M}_{2}=\partial_{t}-\partial_{y}+n \mathbf{E}^{-1},
\end{gathered}
$$

where $\mathbf{E}$ is the shift operator of the discrete variable $n$, defined by $\mathbf{E}^{k} f(n)=f(n+k), \quad k \in Z, t$ and $y$ are continuous variables.

The dressing operators $\mathbf{N}_{1}$ and $\mathbf{N}_{2}$ can be derived from the relations

$$
\begin{array}{r}
\mathbf{N}_{1}\left(\mathbf{I}+\mathbf{K}_{+}(n, m)\right)-\left(\mathbf{I}+\mathbf{K}_{+}(n, m)\right) \mathbf{M}_{1}=0, \\
\mathbf{N}_{2}\left(\mathbf{I}+\mathbf{K}_{+}(n, m)\right)-\left(\mathbf{I}+\mathbf{K}_{+}(n, m)\right) \mathbf{M}_{2}=0 .
\end{array}
$$

Similar to a theorem ref. [7] for continuous systems, it can be proved that $\mathbf{N}_{1}$ and $\mathbf{N}_{2}$ are differential-difference operators. For sake of simplicity, we denote $\mathbf{K}_{+}(n, m)=\mathbf{K}(n, m)$.

We write the dressing operators

$$
\begin{aligned}
& \mathbf{N}_{1}=\mathbf{M}_{1}+\mathbf{D}_{1}, \\
& \mathbf{N}_{2}=\mathbf{M}_{2}+\mathbf{D}_{2} .
\end{aligned}
$$

Acting on function $\varphi_{n}$ on (2.6) and with aid of (2.8), which is reduced to

$$
\begin{aligned}
& M_{1} K(n, m) \varphi_{n}+D_{1} K(n, m) \varphi_{n}+D_{1} \varphi_{n}-K(n, m) M_{1} \varphi_{n} \\
& =\sum_{m=n}^{\infty} K_{t}(n, m) \varphi_{m}+\sum_{m=n}^{\infty} K_{y}(n, m) \varphi_{m}-n \sum_{m=n+1}^{\infty} K(n+1, m) \varphi_{m} \\
& \quad+D_{1} \sum_{m=n}^{\infty} K(n, m) \varphi_{m}+D_{1} \varphi_{n}+\sum_{m=n+1}^{\infty} K(n, m-1) \varphi_{m},
\end{aligned}
$$

from which, comparing coefficient of $\varphi_{n}$, we have

$$
K_{t}(n, n)+K_{y}(n, n)+D_{1} K(n, n)+D_{1}=0 .
$$

Letting $D_{2}=d_{1} E^{-1}$, with aid of (2.7) and (2.9), we have

$$
\begin{aligned}
& M_{2} K(n, m) \varphi_{n}+D_{2} K(n, m) \varphi_{n}+D_{2} \varphi_{n}-K(n, m) M_{2} \varphi_{n} \\
& =\sum_{m=n}^{\infty} K_{t}(n, m) \varphi_{m}-\sum_{m=n}^{\infty} K_{y}(n, m) \varphi_{m}+n \sum_{m=n-1}^{\infty} K(n-1, m) \varphi_{m} \\
& \quad+d_{1} \sum_{m=n-1}^{\infty} K(n-1, m) \varphi_{m}+d_{1} \varphi_{n-1}-n \sum_{m=n-1}^{\infty}(m+1) K(n, m+1) \varphi_{m},
\end{aligned}
$$

from which, comparing coefficient of $\varphi_{n-1}$, we have

$$
n K(n-1, n-1)-n K(n, n)+d_{1} K(n-1, n-1)+d_{1}=0,
$$

and we derive

$$
d_{1}=n \frac{K(n, n)-K(n-1, n-1)}{1+K(n-1, n-1)} .
$$

The following theorem in ref. [7] is an extension of original dressing method, which can yield a wide range of integrable variable-coefficient nonlinear evolution equations. 
Theorem: If the operators $\mathbf{M}_{1}$ and $\mathbf{M}_{2}$ satisfy a relation

$$
\left[\mathbf{M}_{1}, \mathbf{M}_{2}\right]=\rho_{1} \mathbf{M}_{1}+\rho_{2} \mathbf{M}_{2},
$$

where $\rho_{1}, \rho_{2}$ are arbitrary functions of $x, y, n$, then their corresponding dressing operators will satisfy the relation

$$
\left[\mathbf{N}_{1}, \mathbf{N}_{2}\right]=\rho_{1} \mathbf{N}_{1}+\rho_{2} \mathbf{N}_{2} .
$$

Proof: According to (2.6), (2.7) and (2.13), we can give simple proof as follows through simple calculation. In fact,

$$
\begin{aligned}
{\left[\mathbf{N}_{1}, \mathbf{N}_{2}\right]\left(\mathbf{I}+\mathbf{K}_{+}\right) } & =\mathbf{N}_{1}\left(\mathbf{I}+\mathbf{K}_{+}\right) \mathbf{M}_{2}-\mathbf{N}_{2}\left(\mathbf{I}+\mathbf{K}_{+}\right) \mathbf{M}_{1} \\
& =\left(\mathbf{I}+\mathbf{K}_{+}\right) \mathbf{M}_{1} \mathbf{M}_{2}-\left(\mathbf{I}+\mathbf{K}_{+}\right) \mathbf{M}_{2} \mathbf{M}_{1} \\
& =\left(\mathbf{I}+\mathbf{K}_{+}\right)\left[\mathbf{M}_{1}, \mathbf{M}_{2}\right] \\
& =\left(\rho_{1} \mathbf{N}_{1}+\rho_{2} \mathbf{N}_{2}\right)\left(\mathbf{I}+\mathbf{K}_{+}\right) .
\end{aligned}
$$

Actually, variable-coefficient Toda equations are obtained from (2.14). From (2.14), we derived

$$
\begin{gathered}
d_{1 t}+d_{1 y}+\left(n+d_{1}\right)\left(1-E^{-1}\right) D_{1}=0, \\
D_{1 y}-D_{1 t}-n E d_{1}+(n-1) d_{1}=0 .
\end{gathered}
$$

Letting

$$
u_{n}=\frac{1+K(n, n)}{1+K(n-1, n-1)}, \quad D_{1}=v_{n},
$$

then the above Equations (2.15) and (2.16) are reduced to

$$
\begin{gathered}
v_{n, y}-v_{n, t}-\Delta n(n-1)\left(u_{n}-1\right)=0, \\
u_{n, y}+u_{n, t}+u_{n}\left(v_{n}-v_{n-1}\right)=0 .
\end{gathered}
$$

According to (2.19), we assume that

$$
u_{n}=\mathrm{e}^{\chi_{n-1}-\chi_{n}}, \quad v_{n}=\chi_{n, t}+\chi_{n, y},
$$

then (2.18) is reduced to a new integrable variable coefficient Toda equation

$$
\chi_{n, y y}-\chi_{n, t t}-\Delta n(n-1)\left(\mathrm{e}^{\chi_{n-1}-\chi_{n}}-1\right)=0 .
$$

Let $\xi=y+t, \eta=y-t$, then the above equation is reduced to a new $2+1$ dimensional Toda lattice equation

$$
4 \chi_{n, \xi \eta}-\Delta n(n-1)\left(\mathrm{e}^{\chi_{n-1}-\chi_{n}}-1\right)=0 .
$$

The above equations are new and different to classical Toda lattice equation in ref. [17] [18] [19] [20] [21]. Because the coefficient of equation is related to $n$, this is an important physical meaning.

\section{Explicit Solution of Integrable Variable Coefficient Toda Equation}

In this section, we shall use the generalized dressing method to construct explicit solutions of the variable coefficient Toda Equation (2.21). Using the relation $\left[M_{1}, F\right]=0,\left[M_{2}, F\right]=0$, we have 


$$
\begin{aligned}
& F_{t}(n, m)+F_{y}(n, m)-n F(n+1, m)+(m-1) F(n, m-1)=0, \\
& F_{t}(n, m)-F_{y}(n, m)+n F(n-1, m)-(m+1) F(n, m+1)=0 .
\end{aligned}
$$

Assume that (3.1) and (3.2) have $N$-soliton solutions in the form of separation of variables

$$
F(m, n)=\sum_{j=1}^{N} f_{j}(n, t, y) g_{j}(m, t, y),
$$

moveover, we suppose that

$$
K(m, n)=\sum_{j=1}^{N} k_{j}(n, t, y) g_{j}(m, t, y) .
$$

Substituting (3.3) and (3.4) into the GLM (2.3) yields that

$$
K(n, n)=-\left(f_{1}, f_{2}, \cdots, f_{N}\right) L^{-1}\left(g_{1}, g_{2}, \cdots, g_{N}\right)^{\mathrm{T}},
$$

where $L$ is defined by

$$
L_{j l}=\delta_{j l}+\sum_{s=n}^{\infty} g_{j}(t, y, s) f_{l}(t, y, s), \quad 1 \leq j, l \leq N,
$$

and $\delta_{j l}$ is Kronecker's delta.

In what follows, we will obtain one soliton solution of (2.21). First, we give separation of variables solutions for $N=1$ in (3.3) and (3.4),

$$
F(m, n)=f_{1} g_{1}=\mathrm{e}^{p t+q y+n w+\eta_{0}} \mathrm{e}^{m w}, \quad K(m, n)=k_{1} g_{1}=k_{1} \mathrm{e}^{m w} .
$$

From (3.5), we derive

$$
K(n, n)=\frac{\mathrm{e}^{p t+q y+2 n w+\eta_{0}}-\mathrm{e}^{p t+q y+(2 n+2) w+\eta_{0}}+\mathrm{e}^{2 p t+2 q y+4 n w+2 \eta_{0}}}{1-\mathrm{e}^{2 w}},
$$

with $p=c h w, q=-s h w$, using (2.17), we have

$$
u_{n}=\frac{1-\mathrm{e}^{2 w}+\mathrm{e}^{p t+q y+2 n w+\eta_{0}}-\mathrm{e}^{p t+q y+(2 n+2) w+\eta_{0}}+\mathrm{e}^{2 p t+2 q y+4 n w+2 \eta_{0}}}{1-\mathrm{e}^{2 w}+\mathrm{e}^{p t+q y+2(n-1) w+\eta_{0}}-\mathrm{e}^{p t+q y+2 n w+\eta_{0}}+\mathrm{e}^{2 p t+2 q y+4(n-1) w+2 \eta_{0}} .} .
$$

Under transformation $u_{n}=\mathrm{e}^{\chi_{n-1}-\chi_{n}}$, we derive one soliton solution of (2.21)

$$
\chi_{n}=\chi_{0}-\ln \left(u_{1} u_{2} \cdots u_{n}\right) \text {. }
$$

\section{Acknowledgements}

The authors thank the authors of the references. The work described in this paper is supported by National Natural Science Foundation of China.

\section{Data Availability}

The data used to support the findings of this study are available from the corresponding author upon request.

\section{Funding}

The work described in this paper is supported by National Natural Science Foundation of China (Grant No.11301149). 


\section{Authors' Contributions}

Yanan Huang and Ting Su do derivation and calculations. Junhong Yao mainly draw soliton solution picture.

\section{Conflicts of Interest}

There is no competition of interests among authors.

\section{References}

[1] Tian, B. and Gao, Y.T. (1975) Variable-Coefficient Balancing-Act Method and Variable-Coefficient KdV Equation from Fluid Dynamics and Plasma Physics. The European Physical Journal B, 22, 351-360.

[2] Hou, S. and Liu, X.D. (2005) A Numerical Method for Solving Variable Coefficient Elliptic Equation with Interfaces. Journal of Computational Physics, 202, 411-445. https://doi.org/10.1016/j.jcp.2004.07.016

[3] Sun, Z.Y., Gao, Y.T., Liu, Y., et al. (2011) Soliton Management for a Variable-Coefficient Modified Korteweg-De Vries Equation. Physical Review E, Statistical, Nonlinear, and Soft Matter Physics, 84, Article ID: 026606. https://doi.org/10.1103/PhysRevE.84.026606

[4] Yan, Z. (1999) Exact Soliton Solutions of the Variable Coefficient KdV-mKdV Equation with Three Arbitrary Functions. Acta Physica Sinica, 48, 1957-1961. https://doi.org/10.7498/aps.48.1957

[5] Gngr, F. (2002) Symmetries and Invariant Solutions of the Two-Dimensional Variable Coefficient Burgers Equation. Journal of Physics A General Physics, 34, 4313.

[6] Manganaro, N. and Parker, D.F. (1993) Similarity Reductions for Variable-Coefficient Coupled Nonlinear Schrödinger Equations, Journal of Physics A: Mathematical and General, 26, 4093-4106.

[7] Dai, H.H. and Jeffrey, A. (1989) The Inverse Scattering Transforms for Certain Types of Variable-Coefficient KdV Equations. Physics Letters A, 139, 369-372.

[8] Jeffrey, A. and Dai, H.H. (1990) On the Application of a Generalizzed Version of the Dressing Method to the Integration of Variable-Coefficient KdV Equation. Rendiconti di Matematica e delle sue Applicazioni, 10, 439-455.

[9] Su, T., Dai, H.H. and Geng, X.G. (2009) On the Application of a Generalized Dressing Method to the Integration of Variable-Coefficient Coupled Hirota Equations. Journal of Mathematical Physics, 50, 226.

[10] Su, T., Dai, H.H. and Geng, X.G. (2013) A Variable-Coefficient Manakov Model and Its Explicit Solutions through the Generalized Dressing Method. Chinese Physics Letters, 30, Article ID: 060201.

[11] Su, T., Ding, G.H. and Fang, J.Y. (2014) Integrable Variable-Coefficient Coupled Cylindrical NLS Equations and Their Explicit Solutions. Acta Mathematicae Applicatae Sinica, 30, 1017-1024. https://doi.org/10.1007/s10255-014-0439-Z

[12] Dai, H.H. and Su, T. (2010) The Generalized Dressing Method with Applications to the Integration of Variable-Coefficient Toda Equations. Proceedings of the Estonian Academy of Sciences, 59, 293-298.

[13] Ivanov, R., Lyons, T. and Orr, N. (2019) Camassa-Holm Cuspons, Solitons and Their Interactions via the Dressing Method. Journal of Nonlinear Science, 30, 225-260.

[14] Katsinis, D., Mitsoulas, I. and Pastras, G. (2021) The Dressing Method as Non Li- 
near Superposition in Sigma Models. Journal of High Energy Physics, 2021, 1-26.

[15] Dubrovsky, V.G. and Topovsky, A.V. (2020) Multi-Lump Solutions of KP Equation with Integrable Boundary via Dressing Method. Physica D Nonlinear Phenomena, 414, Article ID: 132740.

[16] Nabelek, P.V. and Zakharov, V.E. (2020) Solutions to the Kaup? CBroer System and Its $(2+1)$ Dimensional Integrable Generalization via the Dressing Method. Physica D Nonlinear Phenomena, 409, Article ID: 132478.

[17] Date, E. and Tanaka, S. (1976) Analogue of Inverse Scattering Theory for the Discrete Hill's Equation and Exact Solutions for the Periodic Toda Lattice. Progress of Theoretical and Experimental Physics, 56, 457-465.

[18] Henon, M. (1974) Integrals of the Toda lattice. Physics Review B, 9, 1921-1922.

[19] Berezansky, Y.M. and Gekhtman, M.I. (1990) Inverse Problem of the Spectral Analysis and Non-Abelian Chains of Nonlinear Equations. Ukrainian Mathematical Journal, 42, 645-658.

[20] Ma, W.X. and You, Y.C. (2004) Rational Solutions of the Toda Lattice Equation. Chaos, Solitons, Fractals, 22, 395-406.

[21] Ma, W.X. and Maruno, K.I. (2004) Complexiton Solutions of the Toda Lattice Equaiton in Casoration Form. Journal of Physics A, 343, 219-237. 